\title{
Tratamento cirúrgico da catarata pediátrica
}

\author{
Surgical treatmentofpediatriccataract
}

Jacqueline H. Katina ${ }^{1}$

João Marcelo de Almeida Gusmão Lyra²

Charles Demo Souza ${ }^{2}$

Fernando Cançado Trindade ${ }^{3}$
Trabalho realizado no Serviço de Córnea e Catarata do Hospital São Geraldo da Universidade Federal de Minas Gerais (UFMG).

${ }^{1}$ Doutora em Oftalmologia pela UFMG, Médica Responsável pelo Setor de Catarata Pediátrica do Serviço de Córnea e Catarata do Hospital São Geraldo da UFMG.

${ }^{2}$ Fellows do Serviço de Córnea e Catarata do Hospital São Geraldo da UFMG.

${ }^{3}$ Doutor em Oftalmologia pela UFMG, Professor Adjunto do Departamento de Oftalmologia da UFMG, Chefe do Serviço de Córnea e Catarata do Hospital São Geraldo da UFMG.

Endereço para correspondência: Rua Terra Nova, 30, apto. 901 - Belo Horizonte (MG) CEP 30315-470.

E-mail: jkatina@lineadoro.com.br

Recebido para publicação em 02.02.2001

Aceito para publicação em 30.09.2001

\section{RESUMO}

Objetivo: Descrever as diferentes abordagens dos casos cirúrgicos de catarata pediátrica (CP) em pacientes do Serviço de Córnea e Catarata do Hospital São Geraldo da Universidade Federal de Minas Gerais (UFMG), no período de junho de 1998 a novembro de 2000. Métodos: Foram estudados prospectivamente 37 olhos de 31 crianças. Trinta e cinco olhos $(94,6 \%)$ apresentavam catarata congênita e dois olhos $(5,4 \%)$ catarata traumática. A abordagem cirúrgica variou de acordo com a idade dos pacientes, que foram divididos nos seguintes grupos: Grupo I (abaixo de 1 ano de idade): facectomia por aspiração manual, sem implantação de lente intra-ocular (LIO) com capsulotomia posterior primária, nos primeiros 2 olhos; capsulorrexe posterior primária (CPP) nos demais, e vitrectomia anterior (VA) via límbica em todos. Grupo II: (entre 1 e 5 anos de idade): facectomia por aspiração manual, com implantação de LIO com CPP e VA via límbica. Grupo III: (entre 1 e 5 anos de idade): facectomia por aspiração manual, com implantação de LIO, capsulotomia posterior primária e VA via pars-plana. Grupo IV: (acima de 5 anos de idade): facectomia por aspiração manual, com implantação de LIO sem capsulotomia/CPP e VA. Para confecção da capsulorrexe anterior e posterior (quando indicada), também foi utilizado aparelho de radiofreqüência. A capsulotomia posterior primária era realizada com vitreófago do aparelho de facoemulsificação Universal-Alcon. Resultados: LIO foi implantada em 31 olhos $(83,8 \%)$, sendo que $28(90,3 \%)$ dentro do saco capsular e $3(9,7 \%)$ no sulco ciliar. O seguimento pós-operatório variou entre 2 e 27 meses. No grupo IV, houve opacificação secundária da cápsula posterior em 53,8 \% dos casos ( 7 olhos), sendo estes submetidos a capsulotomia com YAG-laser. Conclusão: O uso da radiofrequiência na realização da capsulorrexe anterior e CPP, assim como a VA melhoraram o resultado cirúrgico da $\mathrm{CP}$.

Descritores: Catarata/congênita; Extração de catarata; Capsulorrexe; Criança

\section{INTRODUÇÃO}

Catarata pediátrica $(\mathrm{CP})$ é causa importante de deficiência visual, com incidência aproximada de $0,4 \%{ }^{(1)}$. Estima-se que existam cerca de 200.000 crianças cegas no mundo devido à catarata bilateral ${ }^{(1)}$.

No Brasil a CP acomete 5,5 a 12\% dos pacientes portadores de visão subnormal ${ }^{(2)}$.

O desenvolvimento de novas técnicas e materiais cirúrgicos têm possibilitado reabilitação visual mais eficiente, que depende principalmente da transparência definitiva do eixo visual ${ }^{(3-5)}$. A opacificação da cápsula posterior ocorre em praticamente $100 \%$ dos casos submetidos à cirurgia, sendo considerada um obstáculo importante, que impede o adequado desenvolvimento visual. 
Admite-se que a capsulorrexe posterior primária (CPP) com vitrectomia anterior (VA) deve ser realizada em todos os pacientes até 5 anos de idade ${ }^{(6)}$, uma vez que a opacificação da cápsula posterior nesta faixa etária pode ser densa e de difícil resolução ${ }^{(6-9)}$.

$\mathrm{O}$ advento de instrumentos automatizados que realizam a capsulotomia circular contínua (capsulorrexe), anterior e posterior $^{(4,7)}$, assim como o aprimoramento de aparelhos de VA, acrescentaram uma nova dimensão à cirurgia da $\mathrm{CP}$ com implantação de lente intra-ocular (LIO).

Em crianças, a cápsula cristaliniana é mais elástica, determinando maior dificuldade no controle da capsulorrexe anterior e posterior, mesmo com a utilização de viscoelástico. A realização da capsulorrexe anterior e posterior com radiofreqüência, inicialmente descrita em 1984, veio facilitar este procedimento. Esse método utiliza corrente de alta frequiência $(500 \mathrm{~Hz})$, para coagular e cortar a cápsula cristaliniana ${ }^{(7)}$.

O presente estudo tem por objetivo descrever as diferentes abordagens dos casos cirúrgicos de CP do Serviço de Córnea e Catarata do Hospital São Geraldo da Universidade Federal de Minas Gerais (HSG-UFMG), no período de junho de 1998 a novembro de 2000.

\section{MÉTODOS}

Foram estudados prospectivamente 37 olhos de 31 crianças submetidas à cirurgia de catarata, no Serviço de Córnea e Catarata do HSG-UFMG no período de junho de 1998 a novembro de 2000.

Trinta e cinco olhos $(94,6 \%)$ apresentaram catarata pediátrica e dois $(5,4 \%)$ traumática.

As indicações para cirurgia foram: catarata total ou parcial com dificuldade de visualização do fundo de olho, acuidade visual inferior à 20/60 (tabela de Snellen) ou inferior à Jaeger 3.

Todas as cirurgias foram realizadas pela mesma cirurgiã (JK).

Anestesia geral foi utilizada em todos os casos.

Foi confeccionado túnel escleral de 5,2 mm de extensão a $1,5 \mathrm{~mm}$ do limbo.

A câmara anterior foi aberta numa extensão de $3 \mathrm{~mm}$ através da incisão tunelizada. Nos primeiros 11 casos, realizou-se capsulorrexe anterior com cistítomo e metilcelulose a 2,5\%. Nos 26 casos restantes, foi utilizado aparelho de radiofreqüência, fabricado pela OERTLI da Suíça para a capsulorrexe anterior e posterior.

Em todos os casos, foi realizada facectomia por aspiração manual através de cânula de Simcoe.

Foram implantadas LIOs de PMMA, peça única, de 12,5 mm de comprimento e óptica de 5 X6 mm, modelo SLIM $^{\circledR}$, quando havia integridade da capsulorrexe anterior. Caso contrário, foi implantada lente de PMMA, três peças, de $14 \mathrm{~mm}$ de comprimento, com óptica de $7 \mathrm{~mm}$ de diâmetro, modelo SK-72 ${ }^{\circledR}$, no saco capsular ou sulco ciliar, ambas fabricadas pela Medipha$\cos$ (Belo Horizonte - MG).

A abordagem da cápsula posterior e a implantação da LIO variou da seguinte forma:
Grupo I (abaixo de 1 ano de idade) - facectomia por aspiração manual, sem implantação de LIO, com capsulotomia posterior primária (vitreófago do aparelho de facoemulsificação Universal - Alcon) nos primeiros 2 olhos; CPP com radiofreqüência nos demais, e VA (vitreófago do aparelho de facoemulsificação Universal - Alcon) por via límbica em todos.

Grupo II (entre 1 e 5 anos de idade) - facectomia por aspiração manual, com implantação de LIO com CPP e VA via límbica: realizada CPP de aproximadamente $4 \mathrm{~mm}$ de diâmetro, feita com radiofrequiência, e VA (vitreófago do aparelho de facoemulsificação Universal - Alcon), seguido de implantação da LIO.

Grupo III (entre 1 e 5 anos de idade) - facectomia por aspiração manual, com implantação de LIO com capsulotomia posterior primária e VA via pars-plana: após facectomia via límbica e implantação da LIO, realizou-se esclerotomia, cuja distância do limbo variou conforme a idade do paciente, como mostra a tabela 1. A seguir, realizou-se capsulotomia posterior primária e VA ambas com vitreófago do aparelho de facoemulsificação Universal - Alcon.

Grupo IV (acima de 5 anos de idade) - facectomia por aspiração manual, com implantação de LIO sem capsulotomia/ CPP e VA.

Em todos os casos, ao final da cirurgia, a substância viscoelástica foi removida, a incisão principal, apesar de ser tunelizada e autoselante, foi suturada com mononylon 10.0. Nos casos com esclerotomia, foi utilizado vicryl 7-0 para sua síntese.

No pós-operatório utilizou-se colírio de tropicamida ou de ciclopentolato, 3 vezes ao dia, e colírio de tobramicina e dexametasona 5 vezes ao dia por uma semana, com redução gradativa até 6 semanas. Em alguns casos, com reação inflamatória maior, foi necessário corticóide sistêmico por período limitado.

Os pacientes foram examinados, rotineiramente, no $1^{\circ}, 4^{\circ}$, $8^{\circ}, 15^{\circ}$ e $30^{\circ}$ dias de pós-operatório.

Capsulotomia posterior secundária com YAG-laser foi realizada nos casos não submetidos à capsulotomia/CPP (grupo IV), nos primeiros 2 meses de pós-operatório, quando a cápsula posterior ainda não estava muito fibrosada, porém opacificada. O YAG-laser também foi utilizado para tratamento de opacificação secundária do eixo visual no grupo III, em casos onde capsulotomia posterior primária e VA foram insuficientes, bem como, em casos (grupos III e IV) onde houve formação de membrana pupilar resistente ao tratamento clínico. A aplicação de YAG-laser em crianças não cooperativas foi realizada sob sedação.

\begin{tabular}{|l|}
\hline $\begin{array}{l}\text { Tabela } 1 . \text { Relação entre idade do paciente e distância do limbo para } \\
\text { realização de esclerotomia para abordagem por via pars-plana }\end{array}$ \\
$\begin{array}{lc}\text { Idade do Paciente } & \text { Distância do Limbo } \\
<1 \text { ano } & 2,0 \mathrm{~mm} \\
1-4 \text { anos } & 2,5 \mathrm{~mm} \\
>4 \text { anos } & 3,0 \mathrm{~mm} \\
\text { Wilson ME; Management of aphakia in childhood(17) }\end{array}$ \\
\hline
\end{tabular}


Depois de estabilizado o quadro clínico, os pacientes foram sistematicamente encaminhados ao Serviço de Visão Subnormal do HSG-UFMG, para reabilitação visual.

\section{RESULTADOS}

A idade média dos pacientes foi de 4 anos (6 meses a 10 anos).

O diagnóstico foi estabelecido em média aos 19,6 meses de idade (primeiro mês de vida a 4 anos) e o tempo decorrido entre a primeira consulta no HSG-UFMG e a cirurgia foi em média de 9,1 meses.

O grupo I foi formado por 6 olhos, o grupo II por 8, o grupo III por 10 e o grupo IV por 13, o que é ilustrado no gráfico 1 que mostra a distribuição dos olhos por grupos, de acordo com a idade do paciente, abordagem da cápsula posterior e vítreo anterior e LIO.

LIO foi implantada em 31 olhos (83,8\%), sendo 28 (90,3\%) no saco capsular e três $(9,7 \%)$ no sulco ciliar (por falta de integridade da capsulorrexe anterior).

O pós-operatório variou entre 2 e 27 meses. As complicações mais observadas foram opacificação da cápsula posterior, em 9 casos $(24,3 \%)$, e membrana pupilar, 9 casos $(24,3 \%)$, seguidas de: pigmentos na superfície da LIO, 2 casos $(5,4 \%)$, formação de sinéquias posteriores, 2 casos $(5,4 \%)$, captura pupilar parcial, 1 caso $(2,7 \%)$ e uveíte severa com descolamento de retina subseqüente, 1 caso $(2,7 \%)$. (Vide gráfico 2 )

O grupo III apresentou opacificação secundária do eixo visual em 2 olhos (20\%) e o grupo IV em 7 olhos $(53,8 \%)$. Nestes 9 casos, houve necessidade de YAG-laser. Os grupos I e II não apresentaram opacidade secundária do eixo visual, conforme mostra o fluxograma 1.

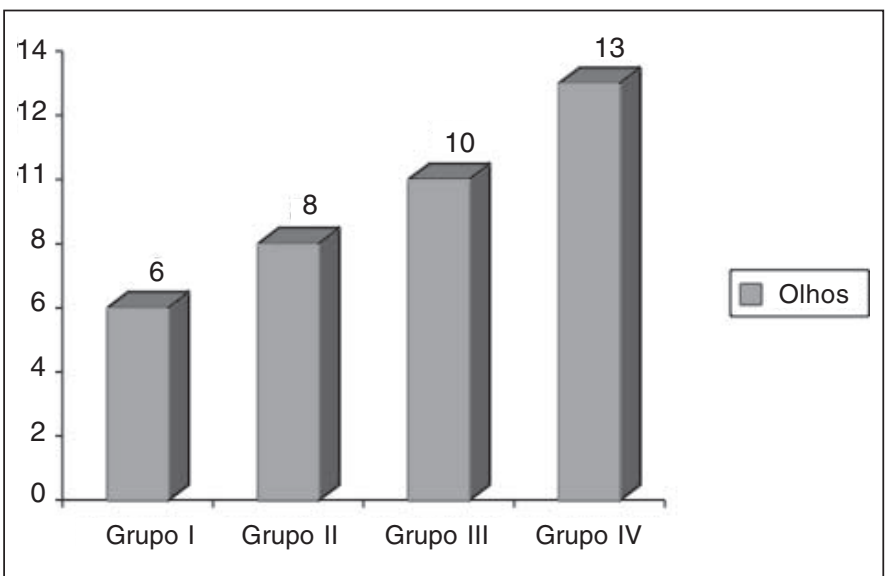

Gráfico 1 - Distribuição dos olhos conforme os grupos de abordagem da cápsula posterior, vítreo anterior e LIO. Grupo I: facectomia por aspiração manual, sem LIO com capsulotomia/CPP e VA via límbica. Grupo II: facectomia por aspiração manual, com LIO com CPP e VA via límbica. Grupo III: facectomia por aspiração manual, com LIO com capsulotomia posterior primária e VA via pars-plana. Grupo IV: facecto

mia por aspiração manual, com LIO sem capsulotomia/CPP e VA

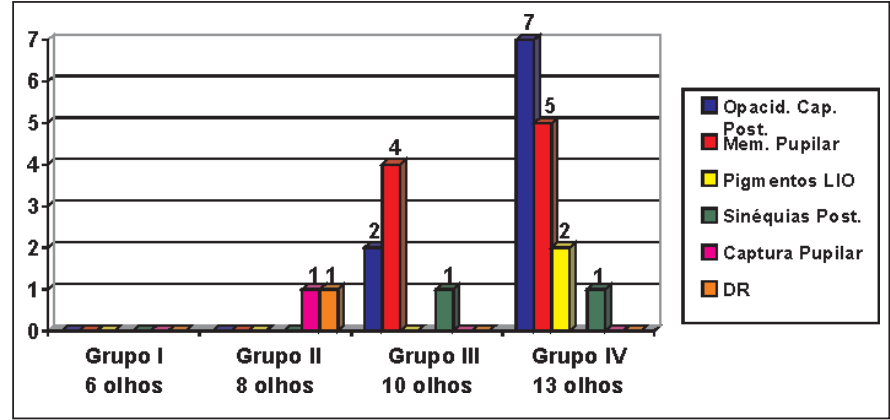

Gráfico 2 - Distribuição das complicações nos grupos de abordagem da cápsula posterior, vítreo anterior e LIO

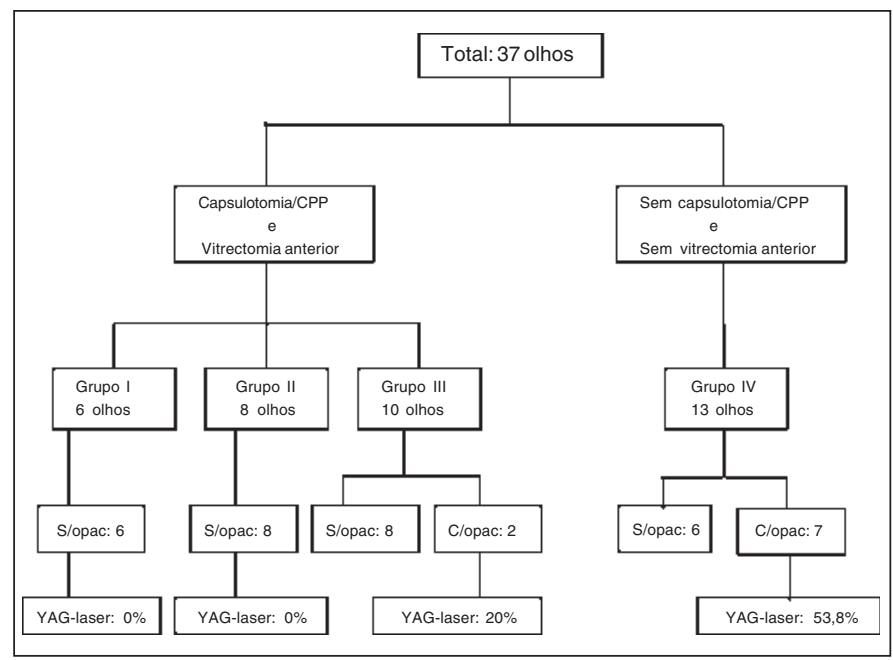

Fluxograma 1

O YAG-laser também foi utilizado para abertura da membrana pupilar, com formação de sinéquias posteriores, resistente ao tratamento clínico, em 7 olhos (18,9\%).

\section{DISCUSSÃO}

O prognóstico visual da cirurgia de $\mathrm{CP}$ depende principalmente da precocidade da intervenção cirúrgica, manutenção do eixo visual transparente e reabilitação visual precoce.

Com relação à indicação cirúrgica da $\mathrm{CP}$, deve-se ressaltar a importância da medida da acuidade visual para perto, pois, esta visão é a mais utilizada pela criança nos primeiros anos de vida e, quando preservada, impede o desenvolvimento da ambliopia. Assim, não existe urgência no tratamento cirúrgico se a criança apresentar moderada redução visual para longe, mas com boa acuidade visual para perto.

No presente estudo, o tratamento cirúrgico da $\mathrm{CP}$ foi realizado numa idade além da desejável, em decorrência, possivelmente, da combinação dos seguintes fatores: diagnóstico tardio, desinformação dos pais e dificuldade de acesso ao serviço de saúde. Observou-se que apenas 16 olhos $(43,2 \%)$ foram 
submetidos à cirurgia antes dos 5 anos de idade, casuística semelhante a descrita por outros autores ${ }^{(9)}$.

A abordagem cirúrgica da CP é variável e controvertida. Deve-se considerar na indicação cirúrgica as desvantagens da correção da afacia com óculos, a inconveniência da utilização de lentes de contato nesta faixa etária e as potenciais complicações da implantação da LIO num olho em crescimento e com maior reatividade tecidual ${ }^{(10-14)}$. Alguns autores chegam mesmo a contra-indicar a implantação de LIO, pelo grande índice de complicações observadas ${ }^{(14)}$.

A determinação do poder dióptrico da LIO a ser implantada é de fundamental importância. Deve-se levar em conta o crescimento ocular ${ }^{(4,11-13)}$. Ao nascimento o comprimento axial é em média $17 \mathrm{~mm}$ e na idade adulta é em torno de $24 \mathrm{~mm}^{(4)}$.

Este crescimento ocorre em 3 fases:

- Primeira fase: até os 2 anos de idade, em média 4,5 mm (90\% do crescimento);

- Segunda fase: até os 6 anos (em média $0,4 \mathrm{~mm}$ por ano);

- Terceira fase: dos 6 anos em diante (em média $1 \mathrm{~mm})^{(4)}$.

A LIO quando implantada precocemente, visando a emetropia, pode resultar tardiamente, em refração miópica acentuada, uma vez que o olho continua crescendo. Altas ametropias no pós-operatório resultam em comprometimento visual se não forem corretamente corrigidas ${ }^{(4,11-13)}$.

Assim sendo, adota-se no Serviço de Córnea e Catarata do HSG-UFMG, como objetivo no cálculo da LIO, a seguinte conduta:

- Até 2 anos de idade: hipocorreção de $20 \%{ }^{(11)}$;

- De 2 a 8 anos de idade: hipocorreção de $10 \%{ }^{(11)}$.

Com a hipocorreção e conseqüente hipermetropia residual, almeja-se, no futuro, alcançar a emetropia com o crescimento fisiológico do globo ocular ${ }^{(4,11-13)}$. Vale enfatizar a necessidade de se corrigir a ametropia pós-operatória residual, no combate à ambliopia.

Com relação às complicações pós-operatórias, a prevalência da opacificação secundária da cápsula posterior mostrou-se, em estudos clínicos, inversamente proporcional à idade ${ }^{(5)}$. Existe uma forte tendência à proliferação de células epiteliais do cristalino na cápsula posterior e/ou na hialóide anterior ${ }^{(5-6,8-9)}$. Segundo trabalhos mais recentes, o índice de opacificação de cápsula posterior em crianças abaixo de 5 anos de idade, aproxima-se de $100 \% \%^{(5-6,8-9,14)}$, constituindo-se num dos maiores obstáculos à reabilitação visual, agravando-se ainda mais a ambliopia. Várias técnicas para evitar a opacificação secundária do eixo visual têm sido relatadas ${ }^{(4,6-9,15-17)}$. Quando não se realiza a capsulotomia/CPP e VA nessa faixa etária, há relatos de até $62,5 \%$ dos pacientes terem necessitado de vitrectomia secundária via pars plana, devido à obstrução densa do eixo visual, resistente à abertura com YAG-laser( ${ }^{(6)}$.

Desta forma, todas as crianças abaixo de 5 anos incluídas no presente estudo, foram submetidas a capsulotomia/CPP e VA. No grupo III, foi realizado YAG-laser devido à opacificação secundária do eixo visual, em 2 olhos, já que a capsulotomia posterior primária e $\mathrm{VA}$ foram insuficientes.
Inicialmente, a capsulotomia posterior primária foi realizada juntamente com a vitrectomia anterior, via pars plana, devido à dificuldade de se fazer a capsulotomia posterior primária por via límbica.

Com o advento da capsulorrexe através da radiofrequência, a abordagem da cápsula posterior por via límbica, tornouse mais fácil e reprodutível, possibilitando-se, assim, efetuar a $\mathrm{CPP}$ e VA por esta via, com maior controle e segurança. A radiofreqüência foi também muito útil nos casos onde havia fibrose ou opacificação densa da cápsula anterior e/ou posterior, obtendo-se assim, uma abertura centralizada e circular.

Contudo, estudos histológicos demonstram diminuição de cerca de $50 \%$ na elasticidade dos bordos da capsulorrexe feita com a radiofrequiência ${ }^{(18-19)}$. Estes fatores, teoricamente, tornariam a borda da capsulorrexe anterior mais vulnerável à ruptura $^{(18-19)}$. Na realidade, em nossa experiência, esta técnica permitiu realizar a capsulorrexe anterior e posterior com facilidade, segurança e previsibilidade, proporcionando assim, condições ideais para a desejável implantação da LIO dentro do saco capsular, objetivo de várias técnicas cirúrgicas ${ }^{(6-8,11-13)}$. Evita-se, dessa forma, o nocivo contato de LIO com tecido uveal, principalmente em se tratando de crianças, com longa expectativa de vida.

Durante a cirurgia, não foram observadas complicações referentes ao uso da radiofreqüência. Ao utilizá-la deve-se preencher a câmara anterior com viscoelástico e ao realizar-se a capsulorrexe, ter o cuidado de não pressionar a ponta da radiofreqüência no cristalino, apenas tocar e delinear continuamente a cápsula anterior e/ou posterior.

Observou-se um número reduzido de complicações pósoperatórias, tais como, captura pupilar, sinéquias posteriores e uveíte, mais freqüentes quando a fixação da LIO foi no sulco ciliar, de acordo com outras publicações ${ }^{(14,20)}$. Além disso, não se evidenciou descentralização da LIO no presente estudo, já que a capsulorrexe anterior e posterior realizadas com radiofreqüência se mantiveram estáveis.

Nos casos onde a capsulorrexe anterior e a posterior foram feitas antes da implantação da LIO, observou-se uma maior dificuldade na inserção desta dentro do saco capsular. No grupo III a técnica cirúrgica foi considerada mais fácil, pois havia mais suporte para a LIO, visto que a VA e capsulotomia posterior primária foram realizadas após a implantação da LIO dentro do saco capsular. Vale citar como alternativa o método de "vitrectorrexis", para realizar a capsulotomia anterior e posterior, utilizando-se a ponteira de vitrectomia anterior, de preferência com bomba Venturi ${ }^{(16)}$.

A partir do segundo semestre de 2000, iniciamos a realização da capsulorrexe posterior com radiofreqüência, pós-implantação da LIO, introduzindo-se a ponteira da caneta de radiofreqüência sob a parte óptica da LIO. A seguir, realizouse a VA via límbica.

A opacificação secundária do eixo visual foi observada em maior proporção no grupo IV, $(53,8 \%)$, sendo necessária apli- 
cação precoce de YAG-laser antes de ocorrer fibrose importante da cápsula posterior, reduzindo-se, dessa forma, o risco de se marcar a LIO, além de permitir abertura adequada da cápsula posterior. O YAG-laser também utilizado para o tratamento de membrana pupilar em 7 olhos (18,9\%), (3 do grupo III e 4 do grupo IV) de um total de 9 (24,3\%) com esta complicação (gráfico 2). Foi empregado em função da má resposta ao tratamento clínico, com formação inicial de sinéquias posteriores, conseqüentes à maior reação inflamatória observada em crianças, podendo, inclusive, desenvolver uveíte fibrinóide ${ }^{(14)}$. Também é importante salientar que nem sempre o tratamento prescrito foi seguido, ocorrendo subseqüentemente maior inflamação no pós-operatório. $\mathrm{O}$ tratamento precoce das membranas pupilares com YAG-laser, pode ter contribuído para um menor índice de sinéquias posteriores, observado neste trabalho, em relação ao descrito em outros estudos ${ }^{(14,17)}$.

Uveíte e descolamento de retina são complicações graves, porém raras após cirurgia de $\mathrm{CP}^{(3-4,14,20)}$. Verificou-se, no presente trabalho, um caso de descolamento de retina (grupo II), em um paciente que apresentou intensa uveíte com formação de traves vítreas, após 2 meses de pós-operatório sem quaisquer intercorrências.

$\mathrm{O}$ tratamento cirúrgico da $\mathrm{CP}$ é ainda um dos maiores desafios da oftalmologia. Contudo, nossos resultados sugerem que a VA e a utilização da radiofrequiência na realização da capsulorrexe anterior e posterior, possibilitaram melhora acentuada na manutenção da transparência do eixo visual, sobretudo em pacientes abaixo de 5 anos de idade, assim como maior controle das complicações pós-operatórias. Entretanto complicações tardias, entre elas o glaucoma, ainda devem ser pesquisadas em período de seguimento posterior.

\section{ABSTRACT}

Purpose: Cases of pediatric cataract were assessed at the Department of Cornea and Cataract of the São Geraldo Eye Hospital, Federal University of Minas Gerais, from June 1998 to November 2000. Methods: Thirty-seven eyes of 31 patients were prospectively analyzed. Thirty-five eyes $(94.6 \%)$ had congenital cataract and 2 eyes (5.4\%) had traumatic cataract. The surgical approach depended on the age of the patients. Patients were classified into the following groups: Group I: (under 1 year of age): manual aspiration of the crystalline lens, with no intraocular lens (IOL) implantation with primary posterior capsulotomy in two eyes; and primary posterior capsulorhexis (PPC) in four eyes and anterior vitrectomy (AV) through limbus in all cases. Group II: (from 1 to 5 years of age): manual aspiration of the crystalline lens with IOL implantation with PPC and AV through limbus. Group III: (from 1 to 5 years of age): manual aspiration of the crystalline lens with IOL implantation with posterior primary capsulotomy and AV through pars-plana. Group IV: (over 5 years of age): manual aspiration of the crystalline lens with IOL implantation without capsulotomy/ PPC and AV. In some cases anterior and posterior capsulorhexis were performed utilizing radiofrequency. The primary posterior capsulotomy was performed utilizing the vitreofagus of the Universal - Alcon phacoemulsificator. Results: IOL implantation was performed in 31 eyes (83.8\%): 28 (90.3\%) in-the-bag implantation and $3(9.7 \%)$ in the ciliary sulcus. The follow-up period varied from 2 to 27 months. In group IV, $53.8 \%$ (7 eyes), had secondary capsular opacification, which underwent YAG laser capsulotomy. Conclusion: Use of the radiofrequency for anterior capsulorhexis and PPC as well as the AV improved the surgical outcome of pediatric cataract.

Keywords: Cataract/congenital; Cataract extraction; Capsulorhexis; Child

\section{REFERÊNCIAS}

1. Foster A, Gilbert C, Rahi J. Epidemiology of cataract in childhood: A global perspective. J Cataract Refract Surg 1997;23(Suppl 1):601-4.

2. Tartarella MB, Kawakami LT, Scarpi MJ, Hayashi S. Aspectos cirúrgicos em catarata congênita. Arq Bras Oftalmol 1995;58:24-8.

3. Zetterström C. Intraocular lens implantation in the pediatric eye. J Cataract Refract Surg 1997;23(Suppl 1):599-600.

4. Wilson ME. Management of aphakia in childhood. Focal Points 1999;17:1-16.

5. Koch DD, Kohnen T. Retrospective comparison of techniques to prevent secondary cataract formation after posterior chamber intraocular lens implantation in infants and children. J Cataract Refract Surg 1997;23(Suppl 1):657-63.

6. Vasavada A, Desai J. Primary posterior capsulorhexis with and without anterior vitrectomy in congenital cataracts. J Cataract Refract Surg 1997;23(Suppl 1):645-51.

7. Klötti R. Bipolar-Nassfeld-Diathermie in der Mikrochirurgie. Klin Monatsbl Augenheilkd 1984;20:658-64.

8. Gimbel HV. Posterior continuous curvilinear capsulorhexis and optic capture of the intraocular lens to prevent secondary opacification in pediatric cataract surgery. J Cataract Refract Surg 1997;23(Suppl 1):652-6.

9. Parks MM. Posterior lens capsulectomy during primary cataract surgery in children. Ophthalmology 1983;90:344-5.

10. Pavlovic S, Jacobi FK, Graef M, Jacobi KW. Silicone intraocular lens implantation in children: preliminary results. J Cataract Refract Surg 2000;26:88-95.

11. Dahan E, Drusedau MU. Choice of lens and dioptric power in pediatric pseudophakia. J Cataract Refract Surg 1997;23(Suppl 1):618-23.

12. Sorkin JA, Lambert SR. Longitudinal changes in axial length in pseudophakic children. J Cataract Refract Surg 1997;23(Suppl 1):624-8.

13. Peterseim MW, Wilson ME. Bilateral intraocular lens implantation in the pediatric population. Ophthalmology 2000;107:1261-6.

14. Sharma N, Pushker N, Dada T, Vajpayee RB, Dada VK. Complications of pediatric cataract surgery and intraocular lens implantation. J Cataract Refract Surg 1999;25:1585-8.

15. Önol M, Özdek SC, Kuksal M, Hasanreisoglu B. Pars plana lensectomy with double-capsule-supported intraocular lens implantation in children. J Cataract Refract Surg 2000;26:486-90.

16. Wilson ME, Bluestein EC, Wang XH, Apple DJ. Comparison of mechanized anterior capsulectomy and manual continuous capsulorhexis in pediatric eyes. J Cataract Refract Surg 1994;20:602-6.

17. Vasavada AR, Trivedi RH. Role of optic capture in congenital cataract and intra-ocular lens surgery in children. J Cataract Refracrt Surg 2000;26:824-31.

18. Butcher JM, Bonshek RD, Raines MF, Kelleher SH. Surgical capsulorhexis and diathermy capsulotomy: an ultrastructural comparison. Eur J Refract Surg 1994;6:344-7.

19. Krag S, Thim K, Corydon L. Diathermic capsulotomy versus capsulorhexis: a biochemical study. J Cataract Refract Surg 1997;23:86-90.

20. Motono M, Tartarella MB, Zim A, Macedo R, Kitadai SS. Resultados de lensectomia em catarata infantil. Arq Bras Oftalmol 1998;61:662-4. 